\title{
Good obstetric outcome in a patient with Segawa disease
}

\author{
Bom resultado obstétrico em uma paciente com doença de Segawa
}

Takashi Watanabe, Shigeki Matsubara

Department of Obstetrics and Gynecology, Jichi Medical University, Tochigi, Japan.

Correspondence: Shigeki Matsubara; Department of Obstetrics and Gynecology, Jichi Medical University; $3311-1$ Yakushiji, Shimotsuke, Tochigi; $329-0498$ Japan;E-mail: matsushi@jichi.ac.jp

Conflict of interest: There is no conflict of interest to declare.

Received 23 January 2012; Received in final form 02 February 2012; Accepted 09 February 2012

\section{Dear Editors,}

This Journal has repeatedly been dealing with Parkinson's disease and its related disorders, in which cerebral dopamine shortage is a considered culprit for the diseases' manifestation. Segawa disease, first described by Japanese pediatric neurologist Dr. Segawa in $1976^{1}$, is an autosomal dominant trait characterized by progressive dystonia with marked diurnal fluctuation. Cerebral dopamine shortage is also its considered culprit. Levodopa improves the neurological symptoms. Carbidopa blocks extracerebral decarboxylation of levodopa and thus increases brain uptake of levodopa. Levodopa monotherapy or combination therapy of levodopa and carbidopa is usually employed ${ }^{1}$. Since Segawa disease manifestation usually starts before reproductive age, neurologists may sometimes be consulted as to pregnancy possibility/outcome or they may have a chance to treat pregnant women associated with this disorder. How pregnancy affects this disorder (or vice-versa) and the appropriate drug selection during pregnancy remains unclear.

We previously reported a primiparous woman with Segawa disease who had a good obstetric outcome ${ }^{2}$. She received levodopa $1,250 \mathrm{mg} /$ day throughout the pregnancy and gave birth to a healthy infant. She was included among the six patients with nine pregnancies that we summarized $^{2}$. Of the six patients, five remained asymptomatic under levodopa treatment, whereas the remaining woman not receiving levodopa showed symptom deterioration with stillbirth. Of the nine pregnancies, levodopa monotherapy was performed in six, all with good obstetric outcomes. The one woman receiving combination therapy had two consecutive miscarriages and, subsequently, received levodopa monotherapy during her third pregnancy, which resulted in a healthy infant ${ }^{4}$. We concluded that during pregnancy levodopa should be continued, and levodopa monotherapy and not combination therapy may be the treatment of choice ${ }^{2}$.

We report here another primiparous woman with Segawa disease. At the age of eight, bilateral clubfeet appeared. Her brother had already been diagnosed as having Segawa disease. With her condition diagnosed as Segawa disease, administration of levodopa $300 \mathrm{mg} /$ day was started, which completely ameliorated her symptoms. After the age of 20, she had taken levodopa 100-300 $\mathrm{mg} /$ day, with the dose modified according to her condition. At the age of 32, she became pregnant and continued to receive levodopa $100-300 \mathrm{mg} /$ day throughout her pregnancy, which well controlled the disease. She vaginally gave term birth to a healthy 3,694 g infant with an Apgar score of 8 at 5 minutes, with an uneventful postpartum course. Levodopa 100-300 mg/day was continued after delivery.

No English or Portuguese reports have been published since our 2009 report $^{3}$. The present patient is the seventh reported case. This successful pregnancy outcome supports our previous proposal of levodopa monotherapy and continuation of levodopa throughout pregnancy and puerperium. Carvidopa increases the levodopa concentration in organs ${ }^{4}$, which may be associated with the miscarriage reported; however, miscarriages during combination therapy may have been coincidental. Further study is needed to confirm our speculation. 


\section{References}

1. Sagawa M, HosakaA, Miyagawa F, Nomura Y, Imai H. Hereditary progressive dystonia with marked diurnal fluctuation. In: Eldridge R, Fahn S (eds). Advances in Neurology. Vol.14. New York: Ravan Press, 1976:215-233.

2. Segawa M, Nomura Y, Nishioka M. Autosomal dominant guanosine triphosphate cyclohydrolase I deficiency (Segawa Disease). Ann Neurol 2003;54:S32-S45.
3. Watanabe T, Matsubara S, Baba Y, Tanaka H, Suzuki T, Suzuki M. Successful management of pregnancy in a patient with Segawa disease: case report and literature review. J Obstet Gynaecol Res 2009;35:562-564.

4. Nomoto M, Kaseda S, Iwata S, Osame M, Fukada T. Levodopa in pregnancy. Mov Disord 1997;12:261.

\section{Dengue and psychiatry disorder}

\section{Dengue e desordens psiquiátricas}

\section{Beuy Joob', Viroj Wiwanitkit ${ }^{2}$}

${ }^{1}$ Sanitation1 Medical, Academic Center, Bangkok, Thailand;

${ }^{2}$ Wiwanitkit House, Bangkhae, Bangkok, Thailand; Visiting University Professor, Hainan Medical University, Haikou, Hainan, China; Adjunct Professor, Joseph Ayo Babalola University, Nigeria.

Correspondence: Beuy Joob; Sanitation1 Medical, Academic Center, Bangkok Thailand; E-mail: beuyjoob@hotmail.com

Conflict of interest: There is no conflict of interest to declare.

Received 06 February 2012; Accepted 14 February 2012

Dear Editors,

The recent publication on dengue and psychiatry disorder is quite interesting ${ }^{1}$. Caixeta et al. ${ }^{1}$ concluded for "relationship between psychiatric disorder and actions upon environmental conditions that favors dengue, as well as its associated public health burden". Indeed, the psychiatric presentation of dengue is possible and considered uncommon ${ }^{2}$. The neurological involvement of dengue can be seen in severe case and this can be the explanation for the psychiatric sequelae ${ }^{3}$. However, for the classical dengue without complication, the relationship between infection and psychiatric manifestation might still be the myth. The actual pathophysiology process has never been clarified although the observation is reported ${ }^{1}$.

\section{References}

1. Caixeta L, Azevedo PV, Caixeta M, Reimer CH. Psychiatry disorders and dengue: Is there a relationship? Arq Neuropsiquiatr 2011;69:920-923.

2. Wiwanitkit V. Dengue fever: diagnosis and treatment. Expert Rev Anti Infect Ther 2010;8:841-845. dengue virus infection. J Neurol Sci 2006;244:117-122. 